\title{
Advances in Multimodal Characterization of Structural Materials
}

\author{
ANDREW T. POLONSKY (iD ${ }^{1,3}$ and AMIT PANDEY ${ }^{2}$ \\ 1.-Materials Mechanics \& Tribology Department, Sandia National \\ Laboratories, Albuquerque, NM 87185, USA. 2.-Digital Transformation (Dx), Lockheed Martin \\ Space, Akron, OH, USA. 3.—e-mail: apolon@sandia.gov
}

The myriad detectors and instruments now available for materials characterization provide researchers with an ever-growing suite of tools to probe material behavior. Progress in the development of instrumentation and workflows that enable the collection, and leverage the potential, of various data modalities have provided novel insights into material behavior. Using data across multiple length scales, or performing complementary analyses of in situ and ex situ data, can help reveal a more complete picture of dynamic processes or material structure. However, the accurate combination, or fusion, of these disparate data modalities presents new challenges. Differences in resolution, as well as the varying length scales at which physical phenomena are exploited to generate these data, necessitate novel approaches to accurately interpret and combine these data. The papers within this special topic focus on the collection and fusion of multimodal data to better understand structural materials. From new frameworks and workflows for data segmentation and analysis, process monitoring, enhancing simulations, or interrogating mechanical response, these papers reveal the potential benefits of utilizing multimodal data.

In "Application of Serial Sectioning to Evaluate the Performance of x-ray Computer Tomography for Quantitative Porosity Measurements in Additively Manufactured Metals," Jolley and colleagues present a correlative study of porosity in three dimensions for an additively manufactured (AM) sample of Ti6-Al-4V using both destructive and nondestructive methods. Comparison of $3 \mathrm{D}$ characterization results with stereological methods showed the applicability of these conventional methods, and

Andrew T. Polonsky and Amit Pandey are Guest Editors for the Advanced Characterization, Testing, and Simulation Committee of TMS and organized the topic Advances in Multi-modal Characterization of Structural Materials in this issue.

(Received August 31, 2021; accepted September 1, 2021;

published online September 23, 2021) correlations between characterization methods revealed the importance of segmentation approaches and resolution differences when quantifying microstructural defects.

In "Towards In-Process Prediction of Voids in Laser Powder Bed Fusion via Data Fusion and Machine Learning," O'Loughlin et al. apply machine learning methodologies to assess defect formation in an AM process from in situ monitoring data. Potential defects were identified from thermal data using clustering approaches on normalized melt pool areas, and kernel smoothing approaches were used to create a defect probability map from segmented computed tomography (CT) data. Correlative analysis was accomplished via a random forest regression, providing a statistical prediction that qualitatively captures porosity from thermal data. Quantitative analysis revealed an ability to reduce false-positive identifications of porosity when using higher-dimensional data.

Donegan et al., in their paper entitled "Multimodal Registration and Fusion of In Situ and Ex Situ Additive Manufacturing Data," demonstrate the utility of a suite of tools designed to combine multiple data streams from the AM process: from part conception and design, in situ sensor data, to ex situ characterization. Using keypoints extracted from each data modality, rigid-body registration of distortion-corrected point cloud data is used to generate a unified volumetric dataset. Such a registration enables correlative analysis of porosity within the part, as well as qualification of geometric deviations from the original design.

In "A Multimodal Data Merging Framework for Correlative Investigation of Strain Localization in Three Dimensions," Charpagne et al. present an approach to combine 3D electron backscattered diffraction (EBSD) and imaging data with 2D high-resolution digital image correlation (HR-DIC) data from in situ mechanical loading experiments, all collected within an electron microscope. Fusion of the data is largely achieved via the use of distortion functions that map coordinates to each 
other in both reference frames. Projection of slip bands identified via HR-DIC into the 3D microstructure in an automated fashion enables statistical analysis of strain localization to better understand micromechanical response.

Nguyen and Rowenhorst, in their paper entitled "Alignment and Fusion of Multimodal 3D Serial Sectioning Datasets," present an automated framework for the fusion of EBSD montage data with backscattered electron (BSE) images as part of a 3D characterization technique. After correcting for distortions and stitching multiple EBSD maps per layer, separate aligned image stacks for both imaging modalities are generated using various signal processing approaches. These two stacks are then aligned using porosity identified in both imaging stacks, enabling higher spatial fidelity in EBSD maps as well as more accurate assessment of porosity within the sample.

$\mathrm{He}$ and his colleagues prepared the paper "Anomalous x-ray Scattering and Extended x-ray Absorption Fine Structure Study of the Local Structure of $\mathrm{CrFeCoNiMo}_{x}(x=0.11,0.18$, and 0.23$)$ highentropy alloys." The authors combine multiple x-ray synchrotron techniques in order to study the local structure of a face-centered-cubic high-entropy alloy (HEA), which lacks long-range chemical ordering. By using multiple characterization approaches, the authors were able to determine that, while Mo atoms are randomly distributed throughout the lattice, there does appear to be short-range ordering in the solid solution, with Mo atoms having a preference to bond with atoms of $\mathrm{Cr}$, which has implications for the deformation behavior of these alloys.

In "Integrating In Situ x-ray Imaging, EnergyDispersive Spectroscopy, and Calculated Phase Diagram Analysis of Solute Segregation during Solidification of an Al-Ag alloy," a large group of researchers present a multiscale, multimodal characterization of an $\mathrm{Al}-\mathrm{Ag}$ alloy. In situ radiography of the melt provides direct visual information on solute segregation during the solidification process, which can be quantitatively understood via ex situ chemical analyses. Applying these data to modeling via calculated phase diagram (CalPhaD) approaches, the authors demonstrate the potential for nondestructive in situ approaches to better understand solidification dynamics.
In "Stress and Deformation Distribution and Microstructure Changes around Pin-Loaded Holes in Medium Manganese Steel Plates," Du and colleagues combine analytical modeling approaches with experimental methods to study the evolution of stress distribution in test specimens of low-carbon, medium-manganese steel plates. Simulation and experiment together reveal the role of microstructure, phase composition, and geometry on deformation behavior. Understanding plastic deformation in this material via experimentally informed analytical methods enables more accurate prediction of the mechanical response of full-scale, real-world components.

In the final contribution of this collection, "Microstructure Quantification and Multiresolution Mechanical Characterization of Ti-Based Bulk Metallic Glass-Matrix Composites," Khosravani, Thadhani, and Kalidindi apply principal component analysis (PCA) on hundreds of high-resolution micrographs in conjunction with indentation stress-strain measurements at the nano- and micro-scale to quantitatively analyze microstructure and mechanical properties of a series of bulk metallic glass-matrix composites. These screening techniques require minimal amounts of material, offering a high-throughput method for understanding process-structure-property relationships in bulk metallic glass-matrix composites more generally.

All titles and authors of the articles are published under the topic "Advances in Multi-modal Characterization of Structural Materials" in the November 2021 issue (vol. 73, no. 11) of JOM. The articles can be accessed fully via the journal's page at: http:// link.springer.com/journal/11837/73/11/page/1

\section{ACKNOWLEDGEMENTS}

Sandia National Laboratories is a multimission laboratory managed and operated by National Technology and Engineering Solutions of Sandia, LLC, a wholly owned subsidiary of Honeywell International, Inc., for the U.S. Department of Energy's National Nuclear Security Administration under Contract DE-NA-0003525.

Publisher's Note Springer Nature remains neutral with regard to jurisdictional claims in published maps and institutional affiliations. 